\title{
On ion temperature gradient and parallel velocity shear instabilities
}

\author{
Andre L. Rogister ${ }^{\mathrm{a})}$ \\ Institut für Plasmaphysik, Forschungszentrum Jülich GmbH, EURATOM-FZJ Association, \\ Trilateral Euregio Cluster, D-52425, Jülich, Germany \\ Raghvendra Singh and Predhiman K. Kaw \\ Institute for Plasma Research, Bhat, Gandhinagar-382 428, India
}

(Received 25 August 2003; accepted 22 January 2004; published online 16 April 2004)

\begin{abstract}
The local dispersion relation for waves with frequencies in the range of the diamagnetic frequencies $\omega_{j}^{*}$ and parallel wave numbers satisfying the conditions $k_{\|} c_{s} / \omega_{e}^{*} \sim 1$ and $q R k_{\|} \gg 1$ has been obtained in the framework of kinetic theory keeping the equilibrium density, temperature, and parallel velocity gradients into account ( $j$ is the species index, $q R$ the connection length, and $c_{s}$ the speed of sound). The analysis applies to the cases where the radial scale of the oscillations is comparable to or smaller than the equilibrium length scale. As the velocity-space integral appearing in the dispersion relation can be calculated only in asymptotic limits, exact instability criteria are obtained by means of the Nyquist diagram. Defining $\tau_{i}=T_{i} / T_{e}, \eta_{i}=\partial_{r} \ln T_{i} / \partial_{r} \ln N_{i}$, and $\zeta$ $=\partial_{r} U_{\|, i} / c_{s} \partial_{r} \ln N_{i}$, it is found that unstable modes appear for $\eta_{i}>1+\sqrt{1-\zeta^{2} /\left(1+\tau_{i}\right)}$ (which agrees with the standard ion temperature gradient instability condition $\eta_{i}>2$ if $\zeta=0$ ) and $0<\eta_{i}$ $<1-\sqrt{1-\zeta^{2} /\left(1+\tau_{i}\right)}$ (the case $\eta_{i}<0$ has not been analyzed), i.e., for $\zeta^{2} \geqslant \eta_{i}\left(2-\eta_{i}\right)\left(1+\tau_{i}\right)$ (which does not agree with the standard parallel velocity shear instability condition $|\zeta|>\sqrt{2}$ if $\eta_{i}$ $=0)$. The center of the unstable range is characterized by the relation $k_{\|} c_{s} / \omega_{e}^{*}=-\zeta / 2\left(1+\tau_{i}\right)$ from which it follows that $q R k_{\|} \gg 1$ is verified if $\left[k_{\beta} a_{s} / 2\left(1+\tau_{i}\right)\right] q R \partial_{r} U_{\|, i} / c_{s} \gg 1\left(k_{\beta}\right.$ is the wave vector component in the direction of the binormal). The oscillations are not tied, under those conditions, to any particular rational surface; the roles of magnetic shear, trapped electrons, ion gyroradius and torus curvature are moreover negligible. The growth/decay rate of the oscillations has been calculated in the neighborhood of marginal (in)stability; the excitation/damping mechanism is (inverse) ion Landau damping. The wave frequency is a function of position so that localization of a wave packet results from a competition between linear growth and distortion (wave breaking in smaller eddies). Applications of the theory include the transition from the edge localized mode-free to enhanced D alpha high confinement regime and intermittency. (C) 2004 American Institute of Physics. [DOI: 10.1063/1.1677177]
\end{abstract}

\section{INTRODUCTION}

We noted recently that the experimental conditions leading to the transition from ELM- (edge localized mode-) free to EDA (enhanced $D \alpha$ ) behavior in ALCATOR C-Mod ${ }^{1-3} \mathrm{H}$ (high confinement)-mode discharges do approximately match those required for onset of the parallel velocity shear (PVS) instability according to D'Angelo's criterion ${ }^{4}$ applied to the pedestal. ${ }^{5}$ Moreover, the mode number of the quasicoherent (QC) mode which appears in the EDA phase is in the range expected for the PVS oscillations that are predicted to become first unstable. The washboard (WB) mode observed in relation to type II ELMs in the Joint European Torus (JET) may be triggered by the same instability. ${ }^{6}$ A simple picture of the driving process has been given by Catto et al. ${ }^{7}$ in the limit $T_{i} / T_{e} \ll 1$. Smith and von Goeler extended the analysis of D'Angelo to include wave-particle resonant interaction. ${ }^{8}$ [In Refs. 4-8, the parallel velocity shear instability was referred to as the Kelvin-Helmholtz, or the parallel velocity shear Kelvin-Helmholtz, or the parallel Kelvin-Helmholtz

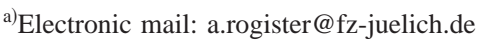

instability; that is confusing since the Kelvin-Helmholtz mode is generally triggered by a radial shear in the perpendicular (usually poloidal) velocity; the Kelvin-Helmholtz appellation is abandoned in this work.]

Motivated by those results, we have undertaken to work out a rigorous theory of

the linear parallel velocity shear instability in the presence of a temperature gradient (which previous theories did not take into account) and its relation to the ion temperature gradient (ITG) instability;

(ii) the nonlinear saturation of the new ITG-PVS instability and the related anomalous particle, momentum and energy fluxes.

As a unique theoretical description cannot encompass all equilibrium situations, the conditions considered are those prevailing at the edge of ALCATOR C-Mod and many other tokamaks, with the exception of the ratio of kinetic to magnetic pressure which is artificially boosted on the ground that the ratio of the amplitudes of the oscillating magnetic and electric fields depends on the magnitudes of the wave vector 
components; although those are ordered here according to the experimental results concerning the QC mode, shorter wavelengths are expected to be generated in nonlinear processes.

The local dispersion relation for ITG-PVS oscillations in the presence of density, temperature, and parallel velocity gradients has been derived from an appropriate expansion of Vlasov's equation. The normalized frequency $z \equiv\left(\omega-\omega_{E}\right.$ $\left.-k_{\|} U_{\|, i}\right) / \omega_{e}^{*}$ (where $\omega_{E}$ is the $\mathbf{E} \times \mathbf{B}$ Doppler frequency, $\omega_{e}^{*}$ the electron diamagnetic frequency, and $U_{\|, i}$ the parallel flow velocity) and the instability criterion depend only on $k_{\|} / k_{\beta}$, the ratio of the wave vector components along the directions of the magnetic field and of the binormal (the binormal unit vector is perpendicular to $\mathbf{B}$ and to the normal to the flux surface). Transition to instability is studied by extending the Nyquist diagram method ${ }^{9}$ employed by Goldston and Rutherford for conventional ITG modes ${ }^{10}$ to situations where $\zeta$ $\equiv \partial_{r} U_{\|, i} / c_{s} \partial_{r} \ln N_{i} \neq 0\left(c_{s}=\sqrt{T_{e} / m_{i}}\right.$ is the sound speed $)$.

It is found that unstable modes appear both for $\eta_{i}>1$ $+\sqrt{1-\zeta^{2} /\left(1+\tau_{i}\right)}$ and $0<\eta_{i}<1-\sqrt{1-\zeta^{2} /\left(1+\tau_{i}\right)}$ (where $\tau_{i}=T_{i} / T_{e}$; the case $\eta_{i}=\partial_{r} \ln T_{i} / \partial_{r} \ln N_{i}<0$ is not discussed). Those inequalities can be recast as $\zeta^{2}>\eta_{i}\left(2-\eta_{i}\right)\left(1+\tau_{i}\right)$. At marginal (in)stability, the mode frequency in the ion rest frame $\omega^{\prime}=\omega-\omega_{E}-k_{\|} U_{\|, i}$ is $\omega^{\prime}=\left(1-\eta_{i} / 2\right) \omega_{e}^{*}$ and the parallel wave number is given by $k_{\|} c_{s} / \omega_{e}^{*}=-\zeta / 2\left(1+\tau_{i}\right)$; it follows that $q R k_{\|}$is much larger than unity $\left(q R k_{\|} \gg 1\right)$ if $\left[k_{\beta} a_{s} / 2\left(1+\tau_{i}\right)\right] q R \partial_{r} U_{\|, i} / c_{s} \gg 1$. We have suggested elsewhere ${ }^{5}$ that the latter condition is met in the H-mode pedestal of ALCATOR C-Mod (and, most likely, of many other tokamaks). The oscillations are not tied, under those conditions, to any particular rational surface. The roles of magnetic shear, trapped electrons, ion gyroradius and torus curvature are moreover negligible so that an analysis in cylindrical geometry is appropriate. We note that our first criterion on $\eta_{i}$ agrees with the standard ion temperature gradient instability condition $\eta_{i}>2$ for $\zeta=0$. Our criterion for $\zeta$ agrees with those of D'Angelo and Catto et al. if $\tau_{i}=1$ and $\eta_{i}=1$ but disagrees if $\eta_{i}=0$ (which is precisely the case they considered); the values of $k_{\|} c_{s} / \omega_{e}^{*}$ differ by a factor 2 from those of D'Angelo; the frequencies $\omega^{\prime}$ fully disagree $\left(\omega^{\prime}=0\right.$ in D'Angelo's paper). A more fundamental difference and the interpretation thereof will be given later.

The growth/decay rate of the oscillations has been calculated in the neighborhood of marginal (in)stability. The excitation/damping mechanism is (inverse) ion Landau damping. Since the radial width of the oscillations we consider is comparable to the equilibrium length scale (the width of the QC mode which is observed in ALCATOR C-Mod EDA discharges is, e.g., comparable to the width of the $\mathrm{H}$-mode pedestal), the wave frequency is a function of position and localization of a wave packet will result from a competition between linear growth and distortion, i.e., wave breaking into smaller eddies. Stabilization and damping by finite $\beta$ effects may act as an energy sink on the shorter wave length modes being generated ( $\beta$ is the ratio of kinetic to magnetic pressure). We recall that inverse Landau damping results from wave-particle resonant interaction. The instability mechanism which is predicted here is therefore quite dif- ferent from that found by D'Angelo in the framework of the two fluids equations, where a bifurcation from two oscillating $\left(\operatorname{Re} \omega^{\prime} \neq 0, \quad \operatorname{Im} \omega^{\prime}=0\right)$ to a damped and a growing $\left(\operatorname{Re} \omega^{\prime}=0, \operatorname{Im} \omega^{\prime} \neq 0\right)$ solutions occurs at instability threshold. The Vlasov-Boltzmann equation on which our analysis is based is a first principle equation; the validity of the two fluids equations relies on certain hypothesis which are not always fulfilled. It is remarkable that the values of $\partial_{r} U_{\|, i} / c_{s} \partial_{r} \ln N_{i}$ and $k_{\|} c_{s} / \omega_{e}^{*}$ obtained at marginal (in)stability from the two approaches are nevertheless comparable.

The paper is organized as follows: In Sec. II, we define and order the dimensionless variables which are relevant to both the equilibrium and the oscillations as powers of a suitable expansion parameter $(\mu)$; the terms in the equilibrium and perturbed Vlasov-Boltzmann equations are ordered accordingly. The Vlasov-Boltzmann equations are solved order by order in Sec. III where we obtain the local dispersion relation; electrostatic theory is here adequate owing to the low kinetic to magnetic pressure ratio $\beta=2 \mu_{0}\left(P_{e}+P_{i}\right) / B^{2}$ and to $k_{\perp} a_{i} \ll 1\left(k_{\perp}=\sqrt{k_{\beta}^{2}-\partial_{r}^{2}}\right.$ is the perpendicular component of the wave vector and $a_{i}$ is the ion gyroradius). Marginal instability is discussed in Sec. IV with the help of the Nyquist diagram. The growth rate near marginal instability is calculated in Sec. V. The relations between the fluctuating magnetic field components and electric potential are obtained in Sec. VI. We conclude in Sec. VII with the summary of the results, the justification of the approximations, and some general remarks. A forthcoming paper will discuss particle, energy, and momentum transport, as well as the ambipolarity constraint.

\section{ORDERING OF DIMENSIONLESS PARAMETERS AND EXPANSION SCHEME}

\section{A. Ordering}

In the pedestals of high density H-mode tokamak discharges, the electron and ion mean free paths along $\mathbf{B}$ are typically of the order of the connection length whereas the equilibrium density and temperature length-scales are comparable to (actually only slightly larger than) the "poloidal ion gyroradius." That implies

$$
\hat{\nu}_{j} \equiv q R \nu_{j} / c_{j} \sim 1
$$

and

$$
\left(a_{i}\right)_{p} /\left|L_{T, N}\right| \sim 1,
$$

where $\nu_{j}$ is the collision frequency for the particular species $\left[\nu_{j}=1 / \tau_{j}\right.$; we adopt Braginskii's definitions of the electron $(j \equiv e)$ and ion $(j \equiv i)$ collision times $\left.{ }^{11}\right], c_{j}=\sqrt{T_{j} / m_{j}}$ is the thermal velocity, $\left(a_{i}\right)_{p}=a_{i}\left|B / B_{\theta}\right|, a_{i}=c_{i} / \Omega_{i}$ is the ion gyroradius, $B_{\theta(\varphi)}$ is the poloidal (the toroidal) component of the magnetic field, and $L_{T}=\left(\partial_{r} \ln T\right)^{-1}, L_{N}=\left(\partial_{r} \ln N\right)^{-1}$.

Since the ratio $B_{\theta} / B_{\varphi}$ is small (typically $\sim 10^{-1}$ ), it is convenient to expand with respect to

$$
\mu=\left|B_{\theta} / B_{\varphi}\right| \ll 1 .
$$


According to neoclassical theory, ${ }^{12,13,5}$ the poloidal and toroidal flow velocities are of order $U_{\theta, i} \approx \partial_{r} T_{i} / e B_{\varphi}$ $\equiv c_{i} a_{i} / L_{T i}$ and $U_{\varphi, i} \approx \partial_{r} T_{i} / e B_{\theta} \equiv c_{i}\left(a_{i}\right)_{p} / L_{T i}$, respectively; we thus introduce [in view of (2) and (3)]:

$$
U_{\beta, i} \sim U_{\theta, i} \sim c_{i} a_{i} / L_{T i} \sim \mu c_{i}
$$

and

$$
U_{\|, i} \sim U_{\varphi, i} \sim c_{i}\left(a_{i}\right)_{p} / L_{T, i} \sim c_{i}
$$

[we define the unit vectors $\hat{n} \equiv \mathbf{B} / B, \hat{b}=\hat{n} \times \hat{p}$, and $\hat{p}$ $=\nabla P /|\nabla P| ; U_{\beta i}=U_{i} \cdot \hat{b}$ is the component of the flow velocity in the direction of the binormal $\hat{b}$; the order of magnitude relations $U_{\beta} \sim U_{\theta}$ and $U_{\|} \sim U_{\varphi}$ follow from $\left.B_{\theta} / B_{\varphi} \sim \mu \ll 1\right]$.

The minor plasma radius is much larger than the edge gradient length scales; thus also the ordering

$$
L_{T(N)} \sim \mu r
$$

moreover

$$
r=\frac{q R B_{\theta}}{B_{\varphi}} \sim \mu q R .
$$

We consider mode structures whose radial length scales are comparable to those of the equilibrium profiles and assume $k_{\beta} \partial_{r} \sim 1$; thus, in view of (2) and (3):

$$
a_{i} \partial_{r} \ln n_{j} \sim a_{i} \partial_{r} \ln p_{j} \sim k_{\beta} a_{i} \sim a_{i} / L_{T(N)} \sim \mu,
$$

where lower-case symbols refer to the oscillations and capitals to the equilibrium variables.

According to D'Angelo, the modes most prone to instability are characterized by $k_{\|} / k_{\beta} \approx a_{i} / L_{N}$ and their angular frequency is $\omega \approx k_{\|} U_{\|, i}-k_{\beta} E_{r} / B$, where $E_{r}$ is the radial electric field. The latter is related to the temperature and density length scales through the radial momentum balance equation

$$
E_{r}=U_{\varphi, i} B_{\theta}-U_{\theta, i} B_{\varphi}+\partial_{r} P_{j} / e N_{i} .
$$

It follows from $(6 a)$ that

$$
k_{\|} a_{i} \sim \mu^{2}
$$

and

$$
\omega \sim \omega_{j}^{*}
$$

where the

$$
\omega_{j}^{*} \equiv k_{\beta}\left(T_{j} / e_{j} B\right) \partial_{r} \ln N_{j}
$$

are the electron and ion diamagnetic frequencies (we consider comparable $T_{e}$ and $T_{i} ; e_{e}=-e_{i}=-e$ ). Moreover, (5a) and $(5 b)$ lead to

$$
k_{\|} q R \sim \mu^{-1} \gg 1
$$

as anticipated.

Relations (1), (6a), (5a), and (5b) imply that the ion collision frequency and the diamagnetic frequencies are in the ratio

$$
\frac{\nu_{i}}{\omega_{i}^{*}} \sim \frac{\nu_{i} L_{N}}{k_{\beta} a_{i} c_{i}} \sim \frac{\hat{\nu}}{k_{\beta} a_{i}} \frac{L_{N}}{q R} \sim \mu
$$

and that

$$
\frac{\omega_{i}^{*}}{\Omega_{i}} \sim k_{\beta} a_{i} \frac{a_{i}}{L_{N}} \sim \mu^{2} .
$$

It remains to scale the ratios $m_{e} / m_{i}$ and $\beta=2 \mu_{0}\left(P_{e}\right.$ $\left.+P_{i}\right) / B^{2}$. It is appropriate to choose

$$
\sqrt{m_{e} / m_{i}} \sim \mu^{2}
$$

and, for reasons mentioned below,

$$
\beta \sim \mu^{2} \text {. }
$$

The edge pedestal of ALCATOR C-Mod is characterized by densities $N_{j} \sim 2 \times 10^{20} \mathrm{~m}^{-3}$ and temperatures $T_{j} \sim 150 \mathrm{eV}$; the magnetic field being $B \sim 5 \mathrm{~T}$, the local value of $\beta$ is $O\left(10^{-3}\right) \ll \sqrt{m_{e} / m_{i}}$. We have nevertheless chosen the ordering (11)-(12) on the grounds that (i) somewhat higher edge values of $\beta$ can occur in tokamaks with lower magnetic fields and (ii) (12) and (6a), respectively, (12) and (11), yield $\left(c / \omega_{p, i}\right) \partial_{r} \ln n_{i} \sim k_{\beta}\left(c / \omega_{p, i}\right) \sim k_{\beta} a_{i} / \sqrt{\beta} \sim 1$, respectively $c_{e}$ $\sim c_{A}$, where $c$ is the speed of light, $\omega_{p, i}$ the ion plasma frequency, and $c_{A}=\sqrt{B^{2} / \mu_{0} m_{i} N_{i}}$ the Alfvén speed. That ordering of $\beta$ will play a role when considering particle transport and the ambipolarity constraint; it has, however, no sizeable effect on the dispersion relation and the stability criterion unless $k_{\perp} a_{i} \sim 1$ (Ref. 14)].

The parallel component of the equilibrium Ampère's equation leads to the scaling relation

$$
r \frac{\partial \ln r B_{\theta}}{\partial r} \sim \beta \frac{q R}{a_{i}} \frac{U_{\|, e}-U_{\|, i}}{c_{i}} .
$$

Since the current flowing through the pedestal is a small fraction of the total plasma current, we request $r \partial_{r} \ln r B_{\theta}$ $\leqslant \mu$ which leads to [in view of (2), (3), (5a), (5b), and (12)]

$$
U_{\|, e}-U_{\|, i} \sim \mu^{2} c_{i}
$$

at most.

\section{B. Expansion scheme}

The ordering of the dimensionless equilibrium parameters and of the frequency and wave vector components of the oscillations allows us to compare the magnitudes of the terms in the corresponding Vlasov-Boltzmann equations once the relation between the amplitudes of the perturbed distribution functions, the electric potential, and the magnetic field components is established.

We first note that the adiabatic relation

$$
e \phi / T_{e} \sim f_{e} / F_{e}
$$

[where $\phi$ is the perturbed electric potential and $f_{e}\left(F_{e}\right)$ the perturbed (equilibrium) electron distribution function] is a straightforward consequence of the inequality $\omega / k_{\|} c_{e}$ $\sim k_{\beta} a_{s} c_{s} / k_{\|} L_{N} c_{e} \sim \mu^{2} \ll 1$ (we note that $\omega_{j}^{*} \sim k_{\beta} a_{s} c_{s} / L_{N}$ ); hence $n_{e} / N_{e} \sim p_{e} / P_{e} \sim e \phi / T_{e}$. The oscillations must be charge neutral since the Debye lengths $\lambda_{D, j}=\sqrt{\varepsilon_{0} T_{j} / N_{j} e^{2}}$ are smaller than the characteristic length-scales by many orders of magnitude [indeed, $k_{\beta} \lambda_{D, j} \sim\left(c_{s} / c\right) k_{\beta} a_{s} / \sqrt{\beta}$ $\left.\sim c_{s} / c\right]$; thus $f_{i} / F_{i} \sim f_{e} / F_{e}$. 
The perturbed magnetic field is obtained from Ampère's equation. The binormal and radial components of the perturbed current are

$$
j_{\beta}=B^{-1} \partial_{r}\left(p_{e}+p_{i}\right)_{\perp}
$$

and

$$
j_{r}=-i k_{\beta} B^{-1}\left(p_{e}+p_{i}\right)_{\perp}
$$

in leading order, where the $p_{\perp}$ are the perpendicular pressures. The parallel component of the perturbed current obtains from $\nabla \cdot \mathbf{j}=0: j_{\|}=-\left(i k_{\|}\right)^{-1} \nabla \cdot \mathbf{j}_{\perp}$. The divergence $\partial_{r} j_{r}+i k_{\beta} j_{\beta}$ of the perpendicular current density vanishes in the leading (see the above relations) and first (as will be shown later) orders. Noting that $k_{\|} / k_{\beta} \sim k_{\|} / \partial_{r} \ln \left(p_{e}+p_{i}\right) \sim \mu$, we obtain $j_{\|} / j_{r}\left(j_{\beta}\right) \sim \mu$. It follows immediately from Ampère's equation that $b_{r} \sim b_{\beta} \sim \mu b_{\|}$with

$$
\begin{aligned}
& b_{\|}^{(0)} / B=-\mu_{0}\left(p_{e}^{(0)}+p_{i}^{(0)}\right)_{\perp} / B^{2}, \\
& \left(\partial_{r}^{2}-k_{\beta}^{2}\right) b_{r}^{(0)}=-\mu_{0} i k_{\beta} j_{\|}^{(0)}-i k_{\|} \partial_{r} b_{\|}^{(0)}, \\
& \left(\partial_{r}^{2}-k_{\beta}^{2}\right) b_{\beta}^{(0)}=\mu_{0} \partial_{r} j_{\|}^{(0)}+k_{\beta} k_{\|} b_{\|}^{(0)},
\end{aligned}
$$

where the notation $(0)$ refers to leading order variables. We thus adopt the ordering

$$
b_{\|} / B \sim \beta p_{\perp} / P \sim \beta(e \phi / T)
$$

and

$$
b_{r} / B \sim b_{\beta} / B \sim \mu \beta p_{\perp} / P \sim \mu \beta(e \phi / T) .
$$

[The perturbed total pressure is thus $\left(p_{e}^{(0)}+p_{i}^{(0)}\right)$ $+B b_{\|}^{(0)} / \mu_{0}=\left(p_{e}^{(0)}+p_{i}^{(0)}\right)_{\|}$in the leading order.]

The kinetic equations describing the small amplitude oscillations

$$
f(r) \exp \left(-i \omega t+i k_{\theta} r \theta+i k_{\varphi} R \varphi\right)
$$

in cylindrical plasmas are given below. [As mentioned in Sec. I, the cylindrical geometry is adequate if $k_{\|} q R \gg 1$; that will be carefully justified in the Sec. VII. We note that $k_{\beta}=\left(B_{\varphi} / B\right)\left(k_{\theta}-B_{\theta} k_{\varphi} / B_{\varphi}\right) \quad$ and $k_{\|}=\left(B_{\varphi} / B\right)\left(k_{\varphi}\right.$ $\left.+B_{\theta} k_{\theta} / B_{\varphi}\right)$.] In order to proceed most easily with the expansion, the order of magnitude of each term with respect to $\Omega_{j} \partial_{s} f_{j}$ (on the right-hand side) is indicated for ions (first estimate) and electrons (second estimate) by appropriate powers of $\mu \ll 1$,

$$
\begin{aligned}
& {\left[\underset{\mu^{2} ; \mu^{6} \mu^{2} ; \mu^{4}}{\left(-i \omega+i k_{\|} v_{\|}+v_{\perp} \cos \varsigma \partial_{r}+i k_{\beta} v_{\perp} \sin \varsigma\right)}+\frac{e_{j}}{m_{j}} E_{r}\left(\cos \varsigma \frac{\partial}{\partial v_{\perp}}-\sin \varsigma \frac{\partial}{\substack{v_{\perp} \partial \varsigma \\
\mu ; \mu^{3}}}\right)\right] f_{j}} \\
& +\frac{B_{\phi} B_{\theta}}{B^{2}}\left(\partial_{r} \ln \frac{B_{\theta}}{B_{\phi}}\right) \frac{v_{\perp}}{2}\left[\sin 2 \varsigma\left(v_{\perp} \frac{\partial}{\partial v_{\|}}-v_{\|} \frac{\partial}{\partial v_{\perp}}\right)-(1+\cos 2 \varsigma) \frac{v_{\|} \partial}{v_{\perp} \partial \varsigma}\right] f_{j} \\
& +\frac{e_{J}}{m_{J}}\left\{\left(-i k_{\|} \phi+i \omega a_{\|}\right) \frac{\partial}{\partial v_{\|}}-\partial_{r} \phi\left(\cos \varsigma \frac{\partial}{\partial v_{\perp}}-\sin \varsigma \frac{\partial}{v_{\perp} \partial \varsigma}\right)-i k_{\beta} \phi\left(\sin \varsigma \frac{\partial}{\partial v_{\perp}}+\cos \varsigma \frac{\partial}{v_{\perp} \partial \varsigma}\right)\right. \\
& \mu^{2} ; \mu^{4} \quad \mu^{4} ; \mu^{6} \quad \mu ; \mu^{3} \\
& \left.-b_{r}\left[\sin \varsigma\left(v_{\perp} \frac{\partial}{\partial v_{\|}}-v_{\|} \frac{\partial}{\partial v_{\perp}}\right)-\cos \varsigma \frac{v_{\|} \partial}{v_{\perp} \partial \varsigma}\right]-b_{\beta}\left[\cos \varsigma\left(v_{\|} \frac{\partial}{\partial v_{\perp}}-v_{\perp} \frac{\partial}{\partial v_{\|}}\right)-\sin \varsigma \frac{v_{\|} \partial}{v_{\perp} \partial \varsigma}\right]\right\}_{J} \\
& \mu^{3} ; \mu^{3} \\
& \mu^{3} ; \mu^{3} \\
& =\Omega_{J} \frac{\partial f_{J}}{\partial \varsigma}+\frac{b_{\|}}{B} \Omega_{J} \frac{\partial F_{J}}{\partial \varsigma}+C_{J}(f, F)+C_{J}(F, f) . \\
& 1 ; 1 \quad \mu^{2} ; \mu^{2} \quad \mu^{3} ; \mu^{5} \quad \mu^{3} ; \mu^{5}
\end{aligned}
$$

The velocity field has been defined by $\mathbf{v}=\hat{p} v_{\perp} \cos s$ $+\hat{b} v_{\perp} \sin \varsigma+\hat{n} v_{\|}$, where $\varsigma$ is the velocity space angle around the equilibrium magnetic field. The gyrofrequency $\Omega_{j}$ $=e_{j} B / m_{j}$ is positive for ions and negative for electrons. No assumption has been made concerning either the equilibrium or the perturbed distribution functions as to their dependence on $\varsigma, v_{\perp}$, and $v_{\|}$. The term proportional to $\partial_{r} \ln \left(B_{\theta} / B_{\phi}\right)$ arises as the unit vectors $\hat{b}=\left(B_{\phi} / B\right) \hat{e}_{\theta}-\left(B_{\theta} / B\right) \hat{e}_{\phi}$ and $\hat{n}$ $=\left(B_{\phi} / B\right) \hat{e}_{\phi}+\left(B_{\theta} / B\right) \hat{e}_{\theta}$ are space-varying; it describes the role of magnetic shear but turns out here to be negligible, in agreement with the findings of Catto et $a{ }^{7}{ }^{7} a_{\|}$is the parallel component of the vector potential. $C_{J}$ is the collision operator for species $j$; it can be split into

$$
C_{e}=C_{e, e}+C_{e, i}
$$

and

$$
C_{i}=C_{i, i}+C_{i, e},
$$

respectively. $C_{e, i}$ and $C_{i, e}$ can be expanded in powers of $\sqrt{m_{e} / m_{i}}$. An important property of the electron-ion and ion-electron collision operators is that 


$$
C_{e, i}(F, F) \sim\left(m_{e} / m_{i}\right)^{1 / 2} \nu_{e e} F_{e}
$$

and

$$
C_{i, e}(F, F) \sim\left(m_{e} / m_{i}\right)^{1 / 2} \nu_{i i} F_{i}
$$

regardless of $F_{i}$, if the departure of the electron distribution function $F_{e}$ from a Maxwellian is not larger than $O\left(\sqrt{m_{e} / m_{i}}\right)^{15}$ and $\left|\mathbf{U}_{e}-\mathbf{U}_{i}\right| \leqslant c_{i}$ (that applies to the full and perturbed operators as well). Nonlinear terms are not considered here, but will be discussed in the forthcoming work on anomalous transport.

Solving Eq. (19) for $f_{J}$ requires knowledge of the equilibrium distribution functions $F_{J}$. The kinetic equations describing the latter include quadratic terms in the oscillation amplitude; an estimate of those is thus required. We shall assume

$$
\frac{e \phi}{T} \sim \mu
$$

which is small compared to the mixing length estimate $\left(e \phi / T \sim 1 / k_{\beta} L_{T(N)} \sim 1\right)$ but leads to a large anomalous ion energy flux $\operatorname{Im}<p_{i} k_{\beta} \phi / B>\sim k_{\beta} a_{i}|e \phi / T|^{2} c_{i} P_{i}$ in comparison to the neoclassical heat flux $q^{2} \nu_{i} a_{i}^{2} P_{i} / L_{T}$ [the ratio being $O\left(\mu^{-1}\right)$; that circumstance will of course simplify the transport analysis in the forthcoming paper]. Under those conditions, the kinetic equations describing the equilibrium distribution functions $F_{j}$ are:

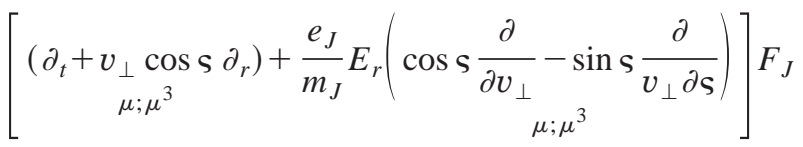

$$
\begin{aligned}
& +\frac{B_{\phi} B_{\theta}}{B^{2}} \partial_{r}\left(\ln \frac{B_{\theta}}{B_{\phi}}\right) \frac{v_{\perp}}{2}\left[\sin 2 \varsigma\left(v_{\perp} \frac{\partial}{\partial v_{\|}}-v_{\|} \frac{\partial}{\partial v_{\perp}}\right)-(1+\cos 2 \varsigma) \frac{v_{\|} \partial}{v_{\perp} ; \mu^{5}}\right] F_{j}
\end{aligned}
$$

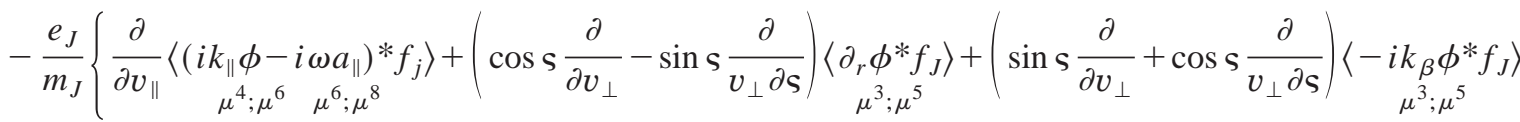

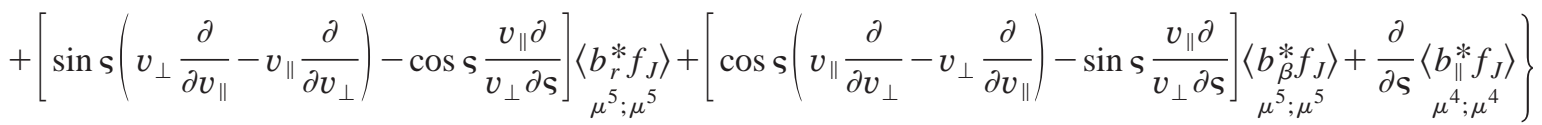

$$
\begin{aligned}
& =C_{J}(F, F)+\left\langle C_{J}\left(f^{*}, f\right)\right\rangle+\Omega_{J} \partial_{\mathrm{S}} F_{J},
\end{aligned}
$$

where a star refers to the complex conjugate and the angular brackets to an average over the oscillations fast time scale (alternatively over the poloidal and toroidal variation of the oscillations).

The evolution and asymptotic forms of the equilibrium and perturbed distribution functions are clearly related. For that reason, the Vlasov-Boltzmann equations (19) and (20) must be solved simultaneously. That is possible only in the framework of a self-consistent expansion, the latter allowing to obtain the higher orders $F_{J}$ and $f_{J}$ in an iterative manner. That justifies the lengthy considerations of this section. The present analysis will show that the distortions of the equilibrium electron and ion distribution functions owing to the fluctuations are small enough as not to affect the dispersion relation and the stability analysis. However, as the leading order perturbed electron distribution is Maxwellian, calculation of anomalous particle transport requires knowledge of higher order perturbed, and therefore equilibrium distribution functions.

\section{ITERATIVE SOLUTION OF THE COUPLED VLASOV-BOLTZMANN EQUATIONS}

In view of the large gyrofrequencies, it will be useful to split $F_{J}$ and $f_{J}$ as

$$
F_{J}=\bar{F}_{J}+\widetilde{F}_{J}, \quad f_{J}=\bar{f}_{J}+\widetilde{f}_{J},
$$

where $\bar{F}_{J}$ and $\bar{f}_{J}$ are the gyrophase averages of $F_{J}$ and $f_{J}$ :

$$
\bar{F}_{J}=\int_{0}^{2 \pi} F_{J} d \varsigma / 2 \pi, \quad \bar{f}_{J}=\int_{0}^{2 \pi} f_{J} d \varsigma / 2 \pi .
$$

The shortest time scale that will be considered in (20) is the energy transport time scale; $\partial_{t} F_{J}$ is accordingly introduced only at the order where the time independent equations have no physically meaningful solution (in the absence of adequate source terms).

\section{A. The leading order equilibrium distribution functions}

Equation (20) yields, at orders $\mu^{0}$ through $\mu^{2}$ :

$$
\widetilde{F}_{i}^{(0)}=0,
$$

$$
\Omega_{i} \partial_{\mathrm{\varsigma}} F_{i}^{(1)}=v_{\perp} \cos \varsigma \partial_{r} F_{i}^{(0)}+\frac{e_{i}}{m_{i}} E_{r} \cos \varsigma \frac{\partial F_{i}^{(0)}}{\partial v_{\perp}},
$$




$$
\begin{aligned}
\Omega_{i} \partial_{\mathrm{\varsigma}} F_{i}^{(2)}= & v_{\perp} \cos \varsigma \partial_{r} F_{i}^{(1)} \\
& +\frac{e_{i}}{m_{i}} E_{r}\left(\cos \varsigma \frac{\partial}{\partial v_{\perp}}-\sin \varsigma \frac{\partial}{v_{\perp} \partial \mathrm{\varsigma}}\right) F_{i}^{(1)},
\end{aligned}
$$

for ions and

$$
\widetilde{F}_{e}^{(0)}=\widetilde{F}_{e}^{(1)}=\widetilde{F}_{e}^{(2)}=0
$$

for electrons. Since (19) yields

$$
\widetilde{f}_{i}^{(0)}=0 \quad \text { and } \quad \widetilde{f}_{e}^{(0)}=\widetilde{f}_{e}^{(1)}=\widetilde{f}_{e}^{(2)}=0,
$$

the gyrophase average of Eq. (20) at order $\mu^{3}$ for ions, respectively, at order $\mu^{5}$ for electrons, reads

$$
\bar{C}_{i}\left(F^{(0)}, F^{(0)}\right)=0 \text {, respectively } \bar{C}_{e}\left(F^{(0)}, F^{(0)}\right)=0 .
$$

(The gyrophase equations are trivially satisfied, and therefore provide no information, at the lower orders.) The leading order equilibrium distribution functions are therefore

$$
F_{i}^{(0)}=N_{i}\left(2 \pi c_{i}^{2}\right)^{-3 / 2} \exp \left[-\frac{v_{\perp}^{2}+\left(v_{\|}-U_{\|, i}\right)^{2}}{2 c_{i}^{2}}\right]
$$

and

$$
F_{e}^{(0)}=N_{e}\left(2 \pi c_{e}^{2}\right)^{-3 / 2} \exp \left[-\frac{v_{\perp}^{2}+\left(v_{\|}-U_{\|, e}\right)^{2}}{2 c_{e}^{2}}\right] .
$$

At this order, there is no constraint on $T_{e}-T_{i}$ and the only constraint on $U_{\|, e}-U_{\|, i}$ is $U_{\|, e}-U_{\|, i} \leqslant \mu c_{e}$. [That follows from the property of the collision operators mentioned below Eq. (19) and, more simply, from the fact that the energy and momentum exchange rates between electrons and ions are smaller than the ion collision frequency by $\sqrt{m_{e} / m_{i}} \sim \mu^{2}$.]

\section{B. The leading order perturbed distribution functions and the dispersion relation}

At order $\mu$ for ions, respectively $\mu^{3}$ for electrons, Eq. (19) yields

$$
\begin{aligned}
\Omega_{i} \partial_{\varsigma} f_{i}^{(1)}= & v_{\perp} \cos \varsigma\left(\partial_{r} f_{i}^{(0)}+\frac{e_{i}}{T_{i}} F_{i}^{(0)} \partial_{r} \phi\right) \\
& +i k_{\beta} v_{\perp} \sin \varsigma\left(f_{i}^{(0)}+\frac{e_{i}}{T_{i}} F_{i}^{(0)} \phi\right) \\
& +\frac{e_{i}}{m_{i}} E_{r} \cos \varsigma \frac{\partial f_{i}^{(0)}}{\partial v_{\perp}}
\end{aligned}
$$

and

$$
\begin{aligned}
\Omega_{e} \partial_{\varsigma} f_{e}^{(3)}= & v_{\perp} \cos \varsigma\left(\partial_{r} f_{e}^{(0)}+\frac{e_{e}}{T_{e}} F_{e}^{(0)} \partial_{r} \phi\right) \\
& +i k_{\beta} v_{\perp} \sin \varsigma\left(f_{e}^{(0)}+\frac{e_{e}}{T_{e}} F_{e}^{(0)} \phi\right) \\
& +\frac{e_{e}}{m_{e}} E_{r} \cos \varsigma \frac{\partial f_{e}^{(0)}}{\partial v_{\perp}} .
\end{aligned}
$$

Those results are introduced into the gyrophase average of Eq. (19) at order $\mu^{2}$ for ions, respectively, at order $\mu^{4}$ for electrons. (The average equations are trivially satisfied at the lower orders.) We obtain

$$
f_{e}^{(0)}=\frac{e \phi}{T_{e}} F_{e}^{(0)}
$$

and

$$
\bar{f}_{i}^{(0)}=\frac{k_{\|}\left(v_{\|}-U_{\|, i}\right) F_{i}^{(0)}-\frac{k_{\beta} T_{i}}{e B} \partial_{r} F_{i}^{(0)}}{\left(\omega-\omega_{E}\right)-k_{\|} v_{\|}} \frac{e \phi}{T_{i}},
$$

where

$$
\begin{gathered}
\frac{k_{\beta} T_{i}}{e B} \partial_{r} F_{i}^{(0)}=\omega_{i}^{*}\left\{1+\eta_{i}\left[\frac{v_{\perp}^{2}+\left(v_{\|}-U_{\|, i}\right)^{2}}{2 c_{i}^{2}}-\frac{3}{2}\right]\right. \\
\left.+\frac{v_{\|}-U_{\|, i}}{c_{i}} \frac{\partial_{r} U_{\|, i}}{c_{i} \partial_{r} \ln N_{i}}\right\} F_{i}^{(0)}, \\
\omega_{E}=-k_{\beta} E_{r} / B
\end{gathered}
$$

is the $\mathbf{E} \times \mathbf{B}$ Doppler frequency.

The expressions of the leading order electron and ion densities are

$$
\begin{aligned}
n_{e}^{(0)}= & \left(e \phi / T_{e}\right) N_{e}, \\
n_{i}^{(0)}= & \left(e \phi / T_{i}\right) \int_{-\infty}^{\infty} d v_{\|}^{\prime}\left(\omega^{\prime}-k_{\|} v_{\|}^{\prime}\right)^{-1}\left\{k_{\|} v_{\|}^{\prime}\right. \\
& \left.-\omega_{i}^{*}\left[1+\eta_{i}\left(\frac{v_{\|}^{\prime 2}}{2 c_{i}^{2}}-\frac{1}{2}\right)+\frac{v_{\|}^{\prime}}{c_{i}} \frac{\partial_{r} U_{\|, i}}{c_{i} \partial_{r} \ln N_{i}}\right]\right\} F_{i}^{(0)},
\end{aligned}
$$

where $v_{\|}^{\prime}=v_{\|}-U_{\|, i}$ and $\omega^{\prime} \equiv \omega-\omega_{E}-k_{\|} U_{\|, i}$. The charge neutrality condition $n_{e}=n_{i}$ provides the wave dispersion relation which thus reads, in the leading order:

$$
1+\tau_{i}=D\left(\omega^{\prime}\right) .
$$

Here $\tau_{i}=T_{i} / T_{e}$ and

$$
\begin{aligned}
D\left(\omega^{\prime}\right)= & N_{i}^{-1} \int_{-\infty}^{\infty} d v_{\|}^{\prime}\left(\omega^{\prime}-k_{\|} v_{\|}^{\prime}\right)^{-1} \\
& \times\left\{\omega^{\prime}-\omega_{i}^{*}\left[1+\eta_{i}\left(\frac{v_{\|}^{\prime 2}}{2 c_{i}^{2}}-\frac{1}{2}\right)+\frac{v_{\|}^{\prime}}{c_{i}} \frac{\partial_{r} U_{\|, i}}{c_{i} \partial_{r} \ln N_{i}}\right]\right\} F_{i}^{(0)} \\
= & \frac{1}{\sqrt{2 \pi \tau_{i}}} \int_{-\infty}^{\infty} d u(z-\xi u)^{-1}\left[z+\tau_{i}\left(1-\frac{\eta_{i}}{2}\right)\right. \\
& \left.+\zeta u+\frac{\eta_{i}}{2} u^{2}\right] \exp \left(-u^{2} / 2 \tau_{i}\right)
\end{aligned}
$$

where $z=\omega^{\prime} / \omega_{e}^{*}, \quad \zeta=\partial_{r} U_{\|, i} / c_{s} \partial_{r} \ln N_{i}$, and $\xi=k_{\|} c_{s} / \omega_{e}^{*}$. For $\left|\operatorname{Im} \omega^{\prime}\right| \ll\left|\operatorname{Re} \omega^{\prime}\right|$, the function $D\left(\omega^{\prime}\right)$ can be rewritten as 


$$
\begin{aligned}
D\left(\omega^{\prime}\right)= & \frac{1}{\sqrt{2 \pi \tau_{i}}} P \int_{-\infty}^{\infty} d u(z-\xi u)^{-1} \\
& \times\left[z+\tau_{i}\left(1-\frac{\eta_{i}}{2}\right)+\zeta u+\frac{\eta_{i}}{2} u^{2}\right] \exp \left(-u^{2} / 2 \tau_{i}\right) \\
& -i \sqrt{\frac{\pi}{2 \tau_{i}}} \frac{1}{|\xi|}\left[z+\tau_{i}\left(1-\frac{\eta_{i}}{2}\right)+\zeta \frac{z}{\xi}+\frac{\eta_{i}}{2} \frac{z^{2}}{\xi^{2}}\right] \\
& \times \exp \left(-z^{2} / 2 \tau_{i} \xi^{2}\right),
\end{aligned}
$$

where $P$ is the Cauchy principal part integral and the imaginary contribution arises from the residue following the prescription of Landau (the latter being imposed by causality). We note that

(i) The dispersion relation (28) involves only two wave related dimensionless parameters, namely, $z$ and $\xi$; since $\omega_{e}^{*} \propto k_{\beta}$, those are proportional to $\omega^{\prime} / k_{\beta}$ and $\omega^{\prime} / k_{\|}$, respectively;

(ii) Although we ordered $\partial_{r} \ln \left(n_{j}, p_{j}, \phi\right) \sim k_{\beta} \sim L_{N(T)}^{-1}$, the radial derivative of the fluctuations does not appear at this order; in other words, the dispersion relation is scalar or local;

(iii) Unlike the derivative of the equilibrium toroidal velocity, the derivative of the radial electric field does not enter Eq. (28); that implies that the stabilizing or destabilizing roles of $\partial_{r} E_{r}$ and $\partial_{r} U_{\|, i}$ are of quite a different nature;

(iv) The wave frequency in the laboratory frame is a function of various inhomogeneous equilibrium parameters; the oscillation will be localized, as a result, to a region where the linear growth balances the wave disintegration process associated with the frequency gradient; ultimately, $\partial_{r} \omega$ (and therefore the equilibrium gradients) will be controlled by anomalous transport, that is equivalent to self-organized criticality.

\section{NYQUIST STABILITY ANALYSIS}

The Nyquist diagram technique allows us to determine the conditions under which the dispersion relation admits unstable solutions. We assume $\omega_{e}^{*}>0$ without loss of generality; instability occurs if $\operatorname{Im} \omega^{\prime}>0$ and, therefore, $\operatorname{Im} z>0$. We let $z$ trace out a closed contour in the complex plane, going from $-\infty$ to $+\infty$ on the real axis and closing anticlockwise on a semicircle at infinity in the upper half-plane. As $z$ traces out that contour, the function $D(z)$ will also trace out some closed contour (the Nyquist contour) in the complex $D$-plane. If the point $D(z)=1+\tau_{i}$ [cf. Eq. (28)] falls in a region encircled by, and lying to the left of, this contour, then the dispersion relation admits a root with $\operatorname{Im} z>0$, i.e., the plasma is unstable.

The semicircle $|z| \rightarrow \infty$ maps into $\lim _{|z| \rightarrow \infty} D(z)=1$ [cf. Eq. (29)]. To analyze the behavior of $D$ at large positive and large negative real values of $z$ we note that Eq. (30) yields

$$
\lim _{z \rightarrow \pm \infty} D=1+\frac{\tau_{i}}{z}-i \sqrt{\frac{\pi}{2 \tau_{i}}} \frac{\eta_{i}}{2} \frac{z^{2}}{|\xi|^{3}} \exp \left(-\frac{z^{2}}{2 \tau_{i} \xi^{2}}\right) .
$$

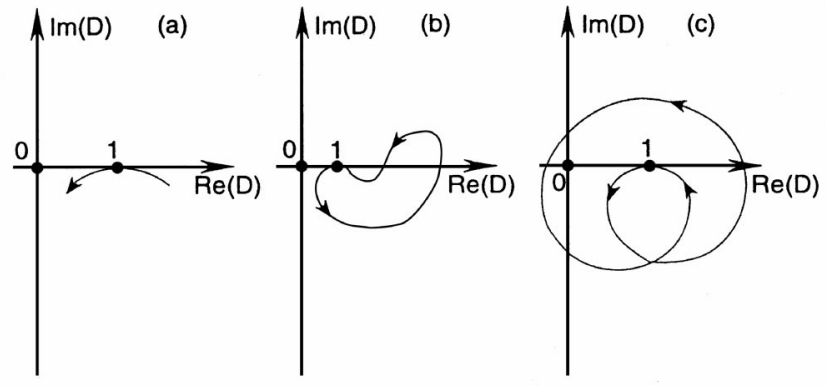

FIG. 1. (a) Map of the semicircle $|z| \rightarrow \infty, \operatorname{Im} z>0$ in the complex $D$ plane; (b) the Nyquist contour for $0<\eta_{i}<2$ and $(\zeta+\xi) / \xi<0$; (c) the Nyquist contour for $\eta_{i}>2$.

Since, on the one hand, $\operatorname{Re} D>1$ for $z \rightarrow+\infty$ and $\operatorname{Re} D<1$ for $z \rightarrow-\infty$ and, on the other hand, $\operatorname{Im} D<0$ for both $z \rightarrow \pm \infty$ (we assume $\eta_{i}>0$ ), $D$ moves in an anticlockwise fashion along a (infinitely small) trajectory which is tangent to the point $D=1$ and otherwise below the real axis as $\operatorname{Re} z$ passes from large and positive values to large and negative values along the semicircle at infinity $(\operatorname{Im} z$ vanishing at the two ends of the contour). The corresponding topology is shown in Fig. 1(a). [We note for completeness that the trajectory in the vicinity of the point $D=1$ is above the real axis if $\eta_{i}$ $<0$ and that $\lim _{z \rightarrow \pm \infty} \operatorname{Im} D$ behaves as $z \exp \left(-z^{2} / 2 \tau_{i} \xi^{2}\right)$, rather than as $z^{2} \exp \left(-z^{2} / 2 \tau_{i} \xi^{2}\right)$, if $\eta_{i}=0$; the case $\eta_{i}=0$ obviously requires a special analysis.] In view of the preceding paragraph, a necessary condition for instability to occur is that the Nyquist $(D)$ contour crosses the real axis as $z$ goes from $-\infty$ to $+\infty$ along the real axis, i.e., that the residue in Eq. (30) vanishes for some values of $z$. Those are solutions of

$$
\frac{\eta_{i}}{2 \xi^{2}} z^{2}+\left(\frac{\zeta}{\xi}+1\right) z+\tau_{i}\left(1-\frac{\eta_{i}}{2}\right)=0
$$

i.e.,

$$
z_{1,2}=-\frac{\xi}{\eta_{i}}(\zeta+\xi)\left[1 \pm \sqrt{1-\frac{\eta_{i} \tau_{i}\left(2-\eta_{i}\right)}{(\zeta+\xi)^{2}}}\right] .
$$

The residue is proportional to the numerator of the integrand in the first term of (30) at $u=z / \xi$. For the values of $z$ which cancel the residue, the numerator of the integrand divides exactly by $(z-\xi u)$. The value of $D$ when $\operatorname{Im} D=0$ can therefore be obtained exactly:

$$
\begin{aligned}
D\left(z_{1,2}\right)= & -\frac{1}{\xi \sqrt{2 \pi \tau_{i}}} \int_{-\infty}^{\infty} d u\left(\frac{\eta_{i}}{2} u+\zeta+\frac{\eta_{i} z_{1,2}}{2 \xi}\right) \\
& \times \exp \left(-u^{2} / 2 \tau_{i}\right) \\
= & -\left(\frac{\zeta}{\xi}+\frac{\eta_{i} z_{1,2}}{2 \xi^{2}}\right) .
\end{aligned}
$$

We note that $D\left(z_{1}\right)>D\left(z_{2}\right)$ if $z_{1}<z_{2}$. That implies that the point $D=1+\tau_{i}$ will lay to the left of, and be encircled by, the Nyquist contour if

$$
D\left(z_{2}\right)<1+\tau_{i}<D\left(z_{1}\right),
$$


where (33) is the sufficient condition for instability. Introducing (31b) into (32) yields

$$
D-1=+\frac{\zeta+\xi}{2 \xi}\left[-1 \pm \sqrt{1-\frac{\eta_{i} \tau_{i}\left(2-\eta_{i}\right)}{(\zeta+\xi)^{2}}}\right] .
$$

The equality $D=1+\tau_{i}$ requests that

$$
\left(1+\tau_{i}\right) \xi^{2}+\zeta \xi+\eta_{i}\left(2-\eta_{i}\right) / 4=0
$$

[whichever sign is considered in $\left.\left(32^{\prime}\right)\right]$ and, therefore,

$$
\xi=\frac{-\zeta \pm \sqrt{\zeta^{2}-\eta_{i}\left(2-\eta_{i}\right)\left(1+\tau_{i}\right)}}{2\left(1+\tau_{i}\right)} .
$$

Different cases have to be envisaged in the framework of the Nyquist stability analysis:

(1) $\eta_{i} \tau_{i}\left(2-\eta_{i}\right)>(\zeta+\xi)^{2}$.

$\operatorname{Im} D=0$ admits no real solution. Therefore, the Nyquist contour cannot cross the real axis. The plasma is stable.

(2) $(\zeta+\xi)^{2}>\eta_{i} \tau_{i}\left(2-\eta_{i}\right)>0$.

$\operatorname{Im} D=0$ admits two real solutions having the same sign. Two subcases have to be considered.

(i) $(\zeta+\xi) / \xi>0$. The two values of $D-1$ given in $\left(32^{\prime}\right)$ are negative and condition (33) cannot be fulfilled. The plasma is stable.

(ii) $(\zeta+\xi) / \xi<0$. The two values of $D-1$ given in $\left(32^{\prime}\right)$ are positive. The Nyquist contour has the form shown in Fig. 1(b). Condition (33) reads

$$
\begin{aligned}
& -\frac{\zeta+\xi}{\xi}\left[1-\sqrt{1-\frac{\eta_{i} \tau_{i}\left(2-\eta_{i}\right)}{(\zeta+\xi)^{2}}}\right] \\
& \quad<2 \tau_{i}<-\frac{\zeta+\xi}{\xi}\left[1+\sqrt{1-\frac{\eta_{i} \tau_{i}\left(2-\eta_{i}\right)}{(\zeta+\xi)^{2}}}\right] .
\end{aligned}
$$

Inspection of Eq. (34) shows that the values of $|\zeta|$ for which these inequalities are satisfied and the plasma is unstable are

$|\zeta|>\left|\zeta_{\text {thr }}\right|=\sqrt{\eta_{i}\left(2-\eta_{i}\right)\left(1+\tau_{i}\right)}$.

To $\zeta=\zeta_{\text {thr }}$ corresponds

$\xi=\xi_{\mathrm{thr}}=-\zeta_{\mathrm{thr}} / 2\left(1+\tau_{i}\right)$.

All other values of $\xi$ are stable when $\zeta=\zeta_{\text {thr }}$. Introducing $\zeta=\zeta_{\text {thr }}$ and $\xi=\xi_{\text {thr }}$ into (35) shows that the equality is satisfied on the left-hand side. The frequency of the marginally unstable mode thus corresponds to the largest of the two values of $z$ in Eq. (31b), namely,

$z=z_{\text {thr }}=1-0.5 \eta_{i}$.

We note that (36) agrees with the criterion of D'Angelo, ${ }^{4}$ namely, $\partial_{r} U_{\|, i}= \pm \sqrt{2} \lambda c_{s}\left[\lambda=-\left(\partial_{r} \ln N_{i}\right)\right]$ if $\tau_{i}=1$ and $\eta_{i}=1 ;\left(36^{\prime}\right)$ and $k_{z} / k_{y}= \pm \lambda c_{s} / \sqrt{2} \Omega_{i}$ then disagrees by a factor 2. There is however complete disagreement if $\eta_{i}=0$ which is the case he actually considered. The disagreement is also complete as concerns the frequency of the marginally stable mode [his equation (18) yields $\omega^{\prime}$ $=0]$. Catto et al. ${ }^{7}$ obtained $\zeta=-\xi\left[1+\tau_{i}+1 / 4 \xi^{2}\right]$ as the marginal stability condition [their local theory, below Eq. (22)]. Minimizing with respect to $\xi$ yields $\zeta_{\text {thr }}$
$= \pm \sqrt{1+\tau_{i}}, \quad-\xi= \pm 1 / 2 \sqrt{1+\tau_{i}}$, and $z=1 / 2$ in agreement with the results of this subsection if and only if $\eta_{i}=1$. The discrepancies are probably related to the following:

(a) The case $\eta_{i}=0$ requires a separate Nyquist analysis (as the contour in the vicinity of the point $D=1$ is no longer below the real axis);

(b) Eq. $\left(36^{\prime}\right)$ yields $\xi_{\text {thr }}=0$ if $\eta_{i}=0$; ion resonant interaction and the instability growth rate $\gamma$ near threshold do vanish faster than any power of $k_{\|} c_{i} / \omega^{\prime}$ $\rightarrow 0$ under those conditions; the Nyquist technique, however, picks up the first mode to become unstable, irrelevant of the expected values of $\gamma$;

(c) Catto et al. did not take ion, but took the electron resonant interaction into account.

Equation (36) yields two unstable domains with respect to $\eta_{i}$; those are

$0<\eta_{i}<1-\sqrt{1-\zeta^{2} /\left(1+\tau_{i}\right)}$

and

$2>\eta_{i}>1+\sqrt{1-\zeta^{2} /\left(1+\tau_{i}\right)}$.

The criterion (38b) agrees with the standard ITG instability condition $\eta_{i}>2$ if $\zeta=0$.

Of interest is the ratio

$\frac{\omega^{\prime 2}}{k_{\|}^{2} c_{i}^{2}}=\frac{z^{2}}{\xi^{2} \tau_{i}}=\frac{\left(1+\tau_{i}\right)\left|2-\eta_{i}\right|}{\tau_{i} \eta_{i}}$

showing that asymptotic expansion of the dispersion function near marginal (in)stability for $\omega^{\prime} / k_{\|} c_{i} \rightarrow 0$ is allowed if $\eta_{i} \rightarrow 2$ and asymptotic expansion for $k_{\|} c_{i} / \omega^{\prime}$ $\rightarrow 0$ is allowed if $\tau_{i} \rightarrow 0$ or $\eta_{i} \rightarrow 0$ (see Appendix). In those cases, resonant interaction of ions with the waves is however exponentially weak.

Of interest is also that $\omega_{\text {thr }}^{\prime}$ has the sign of $\omega_{e}^{*}$ if $\eta_{i}<2$ and the sign of $\omega_{i}^{*}$ if $\eta_{i}>2$.

(3) $\eta_{i}>2$.

$\operatorname{Im} D=0$ admits two real solutions having opposite signs. The corresponding values of $D-1$ have also opposite signs and the Nyquist contour has the form shown in Fig. 1(c). Condition (33) reads

$$
\begin{aligned}
& -\frac{\zeta+\xi}{\xi}\left[\sqrt{\left.1+\frac{\eta_{i} \tau_{i}\left(\eta_{i}-2\right)}{(\zeta+\xi)^{2}}+1\right]}\right. \\
& \quad<2 \tau_{i}<\frac{\zeta+\xi}{\xi}\left[\sqrt{1+\frac{\eta_{i} \tau_{i}\left(\eta_{i}-2\right)}{(\zeta+\xi)^{2}}}-1\right]
\end{aligned}
$$

if $(\zeta+\xi) / \xi>0$ and

$$
\begin{aligned}
& \frac{\zeta+\xi}{\xi}\left[\sqrt{\left.1+\frac{\eta_{i} \tau_{i}\left(\eta_{i}-2\right)}{(\zeta+\xi)^{2}}-1\right]}\right. \\
& \quad<2 \tau_{i}<-\frac{\zeta+\xi}{\xi}\left[\sqrt{1+\frac{\eta_{i} \tau_{i}\left(\eta_{i}-2\right)}{(\zeta+\xi)^{2}}}+1\right]
\end{aligned}
$$


if $(\zeta+\xi) / \xi<0$. The left-hand side inequalities are always satisfied. The right-hand side inequalities are satisfied if $\xi$ lies between the two values given in (34), namely,

$$
\begin{aligned}
- & \frac{\sqrt{\zeta^{2}+\left(1+\tau_{i}\right) \eta_{i}\left(\eta_{i}-2\right)}+\zeta}{2\left(1+\tau_{i}\right)} \\
& <\xi<\frac{\sqrt{\zeta^{2}+\left(1+\tau_{i}\right) \eta_{i}\left(\eta_{i}-2\right)}-\zeta}{2\left(1+\tau_{i}\right)}
\end{aligned}
$$

[it is understood that $\sqrt{\zeta^{2}+\left(1+\tau_{i}\right) \eta_{i}\left(\eta_{i}-2\right)}$ is positive] and $(\zeta+\xi) / \xi>0$, respectively, $(\zeta+\xi) / \xi<0$. Since those conditions are complementary, the plasma is always unstable. The inequality (40) provides the unstable range with respect to $\xi$. Its center is at

$$
\xi=\bar{\xi}=-\zeta / 2\left(1+\tau_{i}\right)
$$

and its width is $\Delta \xi=\sqrt{\zeta^{2}+\left(1+\tau_{i}\right) \eta_{i}\left(\eta_{i}-2\right)} /\left(1+\tau_{i}\right)$; both $|\bar{\xi}|$ and $\Delta \xi$ increase with $|\zeta|$. Assuming $\zeta>0$ in the following discussion, (40) shows that the upper unstable value of $\xi$ is reduced with respect to the case $\zeta=0$ [indeed, $\sqrt{\zeta^{2}+\left(1+\tau_{i}\right) \eta_{i}\left(\eta_{i}-2\right)}-\zeta-\sqrt{\left(1+\tau_{i}\right) \eta_{i}\left(\eta_{i}-2\right)}<0$ ] whereas the lower unstable value decreases by a larger amount than the upper value.

We note that $\left(36^{\prime}\right)$ and (41) will have important consequences concerning anomalous momentum transport.

\section{GROWTH RATE NEAR MARGINAL STABILITY $\left(\eta_{i}<2\right)$}

The dispersion function (29) can be rewritten as

$$
\begin{aligned}
& D(z)= \frac{1}{\sqrt{2 \pi \tau_{i}}} \int_{-\infty}^{\infty} d u \\
& \times\left[\begin{array}{c}
-\frac{1}{\xi}\left(\zeta+\frac{\eta_{i} z}{2 \xi}\right) \\
+\frac{1}{z-\xi u}\left(\frac{\eta_{i} z^{2}}{2 \xi^{2}}+\left(\frac{\zeta}{\xi}+1\right) z+\tau_{i}\left(1-\frac{\eta_{i}}{2}\right)\right] \\
\end{array}\right. \\
& \times \exp \left(-u^{2} / 2 \tau_{i}\right) .
\end{aligned}
$$

[Replace $u$ by $(u-z / \xi)+z / \xi$ in (29) and proceed.] At marginal stability, the second term vanishes [cf. Eq. (31a)] and the first is equal to $1+\tau_{i}$. We let

$$
\zeta=\zeta_{\mathrm{thr}}+\delta \zeta, \quad \xi=\xi_{\mathrm{thr}}+\delta \xi, \quad z=z_{\mathrm{thr}}+\delta z
$$

close to threshold. Expanding the function $D(z)$ and making use of the equalities $\left(36^{\prime}\right)$ and $\eta_{i} z_{\text {thr }} / \xi_{\text {thr }}=-\zeta_{\text {thr }}$ [which follows from (36), (36'), and (37) if $\eta_{i}<2$; the case $\eta_{i}>2$ is not discussed here, since it is always unstable] yields the relation

$$
\begin{aligned}
\left(\frac{\delta z}{z_{\mathrm{thr}}}+\right. & \left.\frac{(\delta \xi)^{2}}{\xi_{\mathrm{thr}}^{2}}-2 \frac{\delta \zeta}{\zeta_{\mathrm{thr}}}\right) \\
= & \left(\frac{1}{1+\tau_{i}} \frac{\delta z}{z_{\mathrm{thr}}}+\frac{(\delta \xi)^{2}}{\xi_{\mathrm{thr}}^{2}}-2 \frac{\delta \zeta}{\zeta_{\mathrm{thr}}}\right) \frac{1}{\sqrt{2 \pi \tau_{i}}} \\
& \times \int_{-\infty}^{\infty} d u \frac{z_{\mathrm{thr}}}{z_{\mathrm{thr}}-\xi_{\mathrm{thr}} u} \exp \left(-u^{2} / 2 \tau_{i}\right)
\end{aligned}
$$

(first order terms in $\delta \xi / \xi_{\text {thr }}$ do vanish since the unstable range is of zero measure with respect to $\xi$ if $\xi=\xi_{\text {thr }}$ ) from which it follows that

$$
\frac{\delta z}{z_{\mathrm{thr}}}=\left(1+\tau_{i}\right)\left(2 \frac{\delta \zeta}{\zeta_{\mathrm{thr}}}-\frac{(\delta \xi)^{2}}{\xi_{\mathrm{thr}}^{2}}\right) \frac{I}{I-\tau_{i}},
$$

where

$$
I=\frac{1}{\sqrt{2 \pi \tau_{i}}} \int_{-\infty}^{\infty} d u \frac{\xi_{\mathrm{thr}} u \exp \left(-u^{2} / 2 \tau_{i}\right)}{z_{\mathrm{thr}}-\xi_{\mathrm{thr}} u} .
$$

Since $z_{\text {thr }}$ is real, we may write

$$
I=\mathfrak{R}-i \mathfrak{I},
$$

where

$$
\mathfrak{R}=\frac{1}{\sqrt{2 \pi \tau_{i}}} P \int_{-\infty}^{\infty} d u \frac{\xi_{\mathrm{thr}} u \exp \left(-u^{2} / 2 \tau_{i}\right)}{z_{\mathrm{thr}}-\xi_{\mathrm{thr}} u}
$$

is the Cauchy principal part integral and

$$
\mathfrak{I}=\sqrt{\pi / 2 \tau_{i}} z_{\mathrm{thr}} \exp \left(-z_{\mathrm{thr}}^{2} / 2 \tau_{i}\right)
$$

is the residue multiplied by the angle $\pi$.

The imaginary part of $\delta z$ can be expressed as

$$
\operatorname{Im} \delta z=\left(1+\tau_{i}\right)\left(2 \frac{\delta \zeta}{\zeta_{\mathrm{thr}}}-\frac{(\delta \xi)^{2}}{\xi_{\mathrm{thr}}^{2}}\right) \frac{z_{\mathrm{thr}} \tau_{i} \mathfrak{I}}{\left(\Re-\tau_{i}\right)^{2}+\mathfrak{I}^{2}},
$$

which shows that $\delta \zeta / \zeta_{\text {thr }}>0$ is destabilizing, whereas both $\delta \xi / \xi_{\text {thr }}>0$ and $\delta \xi / \xi_{\text {thr }}<0$ are stabilizing, as expected from the results of the Nyquist analysis. (We recall that $\delta \omega^{\prime}$ $=\omega_{e}^{*} \delta z$ and $\omega_{e}^{*}>0$ by convention.) The coefficient of $\left[2\left(\delta \zeta / \zeta_{\text {thr }}\right)-\left(\delta \xi / \xi_{\text {thr }}\right)^{2}\right]$ is typically of order unity if $\tau_{i}$ is finite (the standard case for reactor application). Large growth rates are thus predicted (those are proportional to the diamagnetic frequency). The plot $\operatorname{Im} \delta z$ versus $\eta_{i}$ or $\tau_{i}$ is also expected to display a peak when $\mathfrak{R}\left(\eta_{i}, \tau_{i}\right) \rightarrow \tau_{i}$.

In the case (mostly of academic interest) $\tau_{i} \rightarrow 0$, one obtains $I=\mathfrak{R}=\tau_{i} \xi_{\mathrm{thr}}^{2} / z_{\mathrm{thr}}^{2}=\eta_{i} \tau_{i} /\left(2-\eta_{i}\right)$ and $\mathfrak{I}=0$; thus

$$
\lim _{\tau_{i} \rightarrow 0} \frac{\delta z}{z_{\mathrm{thr}}}=\left(\frac{\delta \zeta}{\zeta_{\mathrm{thr}}}-\frac{(\delta \xi)^{2}}{2 \xi_{\mathrm{thr}}^{2}}\right) \frac{\eta_{i}}{\eta_{i}-1} .
$$

That expression diverges for $\eta_{i}=1$. The expansion of the dispersion function $D$ must then be carried out up to second order in $\delta z$. That will yield a quadratic equation whose roots 
will be either real or imaginary. The later case corresponds to an ideal instability, similar to the instability we find in the Appendix for $\tau_{i} \rightarrow 0$ if $|\zeta|>1$. The threshold condition $|\zeta|$ $>1$ is identical to that obtained from the Nyquist analysis [see (36)] if and only if $\eta_{i}=1$.

\section{MAGNETIC FLUCTUATIONS}

Electric field oscillations induce current density and magnetic field oscillations. It is the purpose of this section to obtain the relations between the fluctuations $\mathbf{j}, \mathbf{b}$, and $\phi$.

As already mentioned, the parallel current density obtains from the equation

$$
j_{\|}=-\left(i k_{\|}\right)^{-1} \nabla \cdot \mathbf{j}_{\perp},
$$

where the perpendicular current density can be calculated from

$$
\begin{aligned}
\mathbf{j}_{\perp}= & e\left[\int d \mathbf{v} v_{\perp}(\hat{p} \cos \varsigma+\hat{b} \sin \varsigma) \widetilde{f}_{i}^{(n)}\right. \\
& \left.-\int d \mathbf{v} v_{\perp}(\hat{p} \cos \varsigma+\hat{b} \sin \varsigma) \widetilde{f}_{e}^{(n+2)}\right]
\end{aligned}
$$

at the successive orders. (We note that the electron distribution function of order $n+2$ must be considered simultaneously with the ion distribution function of order $n$ since the characteristic velocities are in the ratio $\sqrt{m_{e} / m_{i}} \sim \mu^{2}$.) $\partial_{\varsigma} f_{i}^{(0)}$ and $\partial_{\varsigma} f_{e}^{(2)}$ vanish identically; with the help of the equations defining $\partial_{\varsigma} f_{i}^{(1)}$ and $\partial_{\varsigma} f_{e}^{(3)}$ (Sec. III B), it is readily verified that

$$
\int d \mathbf{v} v_{\perp}\left(\partial_{r} \cos \varsigma+i k_{\beta} \sin \varsigma\right)\left(\widetilde{f}_{i}^{(1)}-\widetilde{f}_{e}^{(3)}\right)=0 .
$$

$\partial_{\varsigma} f_{i}^{(2)}$ and $\partial_{\varsigma} f_{e}^{(4)}$ are given by

$$
\begin{aligned}
& \left(-i \omega+i k_{\|} v_{\|}\right) f_{i}^{(0)}+\left[v_{\perp}\left(\cos \varsigma \partial_{r}+i k_{\beta} \sin \varsigma\right)\right. \\
& \left.+\frac{e_{i}}{m_{i}} E_{r}\left(\cos \varsigma \frac{\partial}{\partial v_{\perp}}-\sin \varsigma \frac{\partial}{v_{\perp} \partial \varsigma}\right)\right] f_{i}^{(1)} \\
& \quad-\frac{e_{i}}{m_{i}} i k_{\|} \phi \frac{\partial F_{i}^{(0)}}{\partial v_{\|}}-\frac{e_{i}}{m_{i}}\left[\partial_{r} \phi\left(\cos \varsigma \frac{\partial}{\partial v_{\perp}}-\sin \varsigma \frac{\partial}{v_{\perp} \partial \varsigma}\right)\right. \\
& \left.+i k_{\beta} \phi\left(\sin \varsigma \frac{\partial}{\partial v_{\perp}}+\cos \varsigma \frac{\partial}{v_{\perp} \partial \varsigma}\right)\right] F_{i}^{[1]}=\Omega_{i} \frac{\partial f_{i}^{(2)}}{\partial \varsigma}
\end{aligned}
$$

and

$$
\begin{aligned}
i k_{\|} v_{\|} f_{e}^{(0)}+\left[v_{\perp}\left(\cos \varsigma \partial_{r}+i k_{\beta} \sin \varsigma\right)+\frac{e_{e}}{m_{e}} E_{r} \cos \varsigma \frac{\partial}{\partial v_{\perp}}\right] f_{e}^{(1)} \\
-\frac{e_{e}}{m_{e}} i k_{\|} \phi \frac{\partial F_{e}^{(0)}}{\partial v_{\|}}-\frac{e_{e}}{m_{e}}\left(\partial_{r} \phi \cos \varsigma+i k_{\beta} \phi \sin \varsigma\right) \frac{\partial}{\partial v_{\perp}} F_{e}^{[1]} \\
=\Omega_{e} \frac{\partial f_{e}^{(4)}}{\partial \varsigma}
\end{aligned}
$$

respectively; that leads similarly to

$$
\int d \mathbf{v} v_{\perp}\left(\partial_{r} \cos \varsigma+i k_{\beta} \sin \varsigma\right)\left(\widetilde{f}_{i}^{(2)}-\widetilde{f}_{e}^{(4)}\right)=0 .
$$

The parallel component of the oscillating magnetic field obtains from

$$
i k_{\beta} b_{\|}=\mu_{0} j_{r}
$$

(or from $\partial_{r} b_{\|}=-\mu_{0} j_{\beta}$ ) under those conditions. That leads to Eq. (16). We proceed now with the calculation of the divergence of the higher order perpendicular ion and electron currents. We have, at first:

$$
\begin{aligned}
\int d \mathbf{v} v_{\perp}\left(\partial_{r} \cos \varsigma+i k_{\beta} \sin \varsigma\right) f_{i}^{(3)}= & \int d \mathbf{v} v_{\perp}\left(-\partial_{r} \sin \varsigma+i k_{\beta} \cos \varsigma\right) \partial_{\varsigma} f_{i}^{(3)} \\
= & \Omega_{i}^{-1} \int d \mathbf{v}\left[\begin{array}{c}
\left(-i \omega+i k_{\|} v_{\|}\right) v_{\perp}\left(-\partial_{r} \sin \varsigma+i k_{\beta} \cos \varsigma\right) f_{i}^{(1)} \\
-\left[\left(v_{\perp}^{2} / 2\right)\left(\partial_{r}^{2}+k_{\beta}^{2}\right) \sin 2 \varsigma-v_{\perp}^{2} i k_{\beta} \partial_{r} \cos 2 \varsigma\right] f_{i}^{2}
\end{array}\right] \\
& +i k_{\beta} B^{-1}\left(-\phi \partial_{r} N_{i}^{(2)}-E_{r} n_{i}^{(2)}\right) .
\end{aligned}
$$

Integrating by parts and replacing $\partial_{\mathrm{S}} f_{i}^{(2)}$ and $\partial_{\mathrm{S}} f_{i}^{(1)}$, we find after some lengthy but straightforward algebra that

$$
\int d \mathbf{v} v_{\perp}\left(\partial_{r} \cos \varsigma+i k_{\beta} \sin \varsigma\right) f_{i}^{(3)}=i k_{\beta} B^{-1} \Omega_{i}^{-1}\left\{\begin{array}{c}
{\left[\left(\partial_{r}^{2}-k_{\beta}^{2}\right) \phi\right]\left[\left(\omega^{\prime} / k_{\beta}\right) N_{i}-\partial_{r} P_{i} / e B\right]} \\
+\left(\partial_{r} \phi\right) \partial_{r}\left[\left(\omega^{\prime} / k_{\beta}\right) N_{i}+\partial_{r} P_{i} / e B\right] \\
+\partial_{r}\left[\left(\partial_{r} \omega_{E} / k_{\beta}\right)\left(N_{i} \phi+p_{\perp, i} / e\right)\right]
\end{array}\right\}-i k_{\beta} B^{-1} E_{r} n_{i}^{(2)},
$$

where

$$
\begin{aligned}
p_{\perp, i} & =\int d \mathbf{v}\left(m_{i} v_{\perp}^{2} / 2\right) f_{i}^{(0)} \\
& =n_{i}^{(0)} T_{i}\left[1+\frac{\eta_{i} \omega_{e}^{*}}{\sqrt{2 \pi} c_{i}} \int_{-\infty}^{\infty} d u \frac{\exp \left(-u^{2} / 2 c_{i}^{2}\right)}{\omega^{\prime}-k_{\|} u}\right] .
\end{aligned}
$$

(The first and second order equilibrium density $N_{i}^{(1)}$ and $N_{i}^{(2)}$ have been absorbed in $N_{i}^{(0)} \equiv N_{i}$.) Furthermore,

$$
\int d \mathbf{v} v_{\perp}\left(\partial_{r} \cos \varsigma+i k_{\beta} \sin \varsigma\right) f_{e}^{(5)}=-i k_{\beta} B^{-1} E_{r} n_{e}^{(2)}
$$

The parallel current density is therefore given by the expression 


$$
\begin{aligned}
j_{\|}= & -\left(k_{\beta} / k_{\|}\right)\left(m_{i} / B^{2}\right) \\
& \times\left\{\begin{array}{c}
{\left[\left(\partial_{r}^{2}-k_{\beta}^{2}\right) \phi\right]\left[\left(\omega^{\prime} / k_{\beta}\right) N_{i}-\partial_{r} P_{i} / e B\right]} \\
+\left(\partial_{r} \phi\right) \partial_{r}\left[\left(\omega^{\prime} / k_{\beta}\right) N_{i}+\partial_{r} P_{i} / e B\right] \\
+\partial_{r}\left[\left(\partial_{r} \omega_{E} / k_{\beta}\right)\left(N_{i} \phi+p_{\perp, i} / e\right)\right]
\end{array}\right\} .
\end{aligned}
$$

Equation (50) shows that the parallel current arises as to cancel the charge separation which tends to occur in the plane perpendicular to $\mathbf{B}$ owing to finite ion gyroradius effects. The radial and binormal components of the perturbed magnetic field are obtained from

$$
\left(\partial_{r}^{2}-k_{\beta}^{2}\right) b_{r}=-\mu_{0} i k_{\beta} j_{\|}-i k_{\|} \partial_{r} b_{\|}
$$

and

$$
\left(\partial_{r}^{2}-k_{\beta}^{2}\right) b_{\beta}=\mu_{0} \partial_{r} j_{\|}+k_{\beta} k_{\|} b_{\|},
$$

respectively. Thus,

$$
\begin{aligned}
& \left(\partial_{r}^{2}-k_{\beta}^{2}\right) b_{r} \\
& =\frac{i k_{\beta}^{2}}{k_{\|} N_{i} c_{A}^{2}}\left\{\begin{array}{c}
{\left[\left(\partial_{r}^{2}-k_{\beta}^{2}\right) \phi\right]\left[\left(\omega^{\prime} / k_{\beta}\right) N_{i}-\partial_{r} P_{i} / e B\right]} \\
+\left(\partial_{r} \phi\right) \partial_{r}\left[\left(\omega^{\prime} / k_{\beta}\right) N_{i}+\partial_{r} P_{i} / e B\right] \\
+\partial_{r}\left[\left(\frac{\partial_{r} \omega_{E}}{k_{\beta}}+\frac{k_{\|}^{2}}{k_{\beta}^{2}} \Omega_{i}\right)\left(\frac{p_{\perp, e}^{(0)}+p_{\perp, i}^{(0)}}{e}\right)\right]
\end{array}\right\}
\end{aligned}
$$

and

$$
\begin{aligned}
& \left(\partial_{r}^{2}-k_{\beta}^{2}\right) b_{\beta} \\
& =-\frac{k_{\beta}}{k_{\|} N_{i} c_{A}^{2}} \\
& \quad \times \partial_{r}\left\{\begin{array}{c}
{\left[\left(\partial_{r}^{2}-k_{\beta}^{2}\right) \phi\right]\left[\left(\omega^{\prime} / k_{\beta}\right) N_{i}-\partial_{r} P_{i} / e B\right]} \\
+\left(\partial_{r} \phi\right) \partial_{r}\left[\left(\omega^{\prime} / k_{\beta}\right) N_{i}+\partial_{r} P_{i} / e B\right] \\
+\partial_{r}\left[\left(\frac{\partial_{r} \omega_{E}}{k_{\beta}}+\frac{k_{\|}^{2}}{\partial_{r}^{2}} \Omega_{i}\right)\left(\frac{p_{e, \perp}^{(0)}+p_{\perp, i}^{(0)}}{e}\right)\right]
\end{array}\right\} .
\end{aligned}
$$

We have made use of $p_{\perp, e}^{(0)}=p_{\|_{\hat{\rho}} e}^{(0)}=p_{e}^{(0)}=e N_{e} \phi$ and introduced the symbolic notation $1 / \partial_{r}^{2}$. It is easily seen that the normalized magnetic and electric fluctuations $b_{r} / B$ and $e \phi / T$ are in the ratio

$$
\frac{b_{r} / B}{e \phi / T} \approx \beta \frac{k_{\beta}^{2} a_{i}^{2}}{k_{\|} L_{N(T)}}
$$

or

$$
\frac{b_{r} / B}{e \phi / T} \approx \beta \frac{k_{\|} \partial_{r}}{\partial_{r}^{2}-k_{\beta}^{2}}
$$

according to whether the first or the last term on the righthand side of (51) is dominant.

\section{DISCUSSION}

\section{A. Summary of the results}

In this paper, we have obtained and analyzed the local kinetic dispersion relation for oscillations with frequencies and perpendicular, respectively parallel, wave numbers in the following ranges: $\omega \sim \omega_{j}^{*}\left(\omega_{j}^{*}\right.$ is the electron or ion diamagnetic frequency; we assume $T_{i} \sim T_{e}$ so that $\left.\omega_{i}^{*} \sim-\omega_{e}^{*}\right)$, $k_{\beta} a_{i} \sim a_{i} \partial_{r} \ln n_{j} \sim \mu \ll 1\left(a_{i}\right.$ is the ion gyro-radius and $\mu \ll 1$ an expansion parameter of order $B_{\theta} / B_{\varphi}$ ) and $k_{\|}$ $\sim k_{\beta} a_{i} / L_{N}$, where $L_{N}=\partial_{r} \ln N_{j}$. Unlike previous analysis, ${ }^{4,7,10}$ we have taken the equilibrium density, temperature, and parallel velocity gradients simultaneously into account (parameters $\eta_{i}=\partial_{r} \ln T_{i} / \partial_{r} \ln N_{i} \sim 1 \quad$ and $\zeta$ $\left.=\partial_{r} U_{\|, i} / c_{s} \partial_{r} \ln N_{i} \sim 1\right)$; we have assumed that $L_{N} \ll r \ll q R$, as is typical for transport barriers and, in particular, for H-mode pedestals; we have also neglected the torus curvature and considered the electrons to be "adiabatic." Those simplifications and the local analysis are justified below for $k_{\|} q R \gg 1$, which inequality turns out to be the verified for the marginally unstable oscillations in the $\mathrm{H}$-mode pedestal of ALCATOR C-Mod if the parallel velocity gradient is estimated from neoclassical theory. ${ }^{5,13}$ As a consequence, the dispersion relation (30) involves only two parameters defining the oscillations, namely, $\omega^{\prime} / k_{\beta}$ and $\omega^{\prime} / k_{\|}$.

Our primary aim has been to determine the exact stability limits in the parameter space $\eta_{i}, \zeta$, and $\tau_{i}=T_{i} / T_{e}$. Those cannot be obtained from asymptotic expansions of the dispersion relation (29) or (30), the reason being that the asymptotic regime to consider is not known a priori and may moreover change with the values of the parameters $\tau_{i}$ or $\eta_{i}$. [As Eq. (39) shows, expansion with respect to $k_{\|} v_{\|}^{\prime} / \omega^{\prime}$ is e.g. not allowed if $\eta_{i} \rightarrow 2$ (hence $\omega_{\mathrm{thr}}^{\prime} \rightarrow 0$ ) but is possible if $\eta_{i} \rightarrow 0$ (hence $\omega_{\mathrm{thr}}^{\prime} \rightarrow \omega_{e}^{*}$ ). The first, respectively the second, case corresponds to ITG, respectively electron drift branch, marginal (in)stability if $\zeta \rightarrow 0$; generally speaking, expansion of the dispersion function leads to misleading results for the ITG-PVS instability.] The Nyquist diagram technique is the only analytical method that is able to yield accurate stability criteria under those conditions. The dispersion function however can be simplified near threshold, once the threshold relation is known, and the expression of the growth rate be obtained. Instability is driven by wave-particle resonant interaction (more precisely by inverse ion Landau damping), except in the limit $\tau_{i} \rightarrow 0$ which, for fusion plasmas, is of no interest. Despite the large gradients, the electrostatic approximation of the dispersion relation is justified for the plasma edge.

More details on the results obtained in the present work are given hereafter.

(1) The local instability condition reads

$$
\zeta^{2} \geqslant \zeta_{\mathrm{thr}}^{2}=\eta_{i}\left(2-\eta_{i}\right)\left(1+\tau_{i}\right)
$$

[That result disagrees with those obtained by D'Angelo and by Catto et al. for the parallel velocity shear instability $\left(|\zeta|>\sqrt{1+\tau_{i}}\right.$ for $\left.\eta_{i}=0\right)$; the case $\eta_{i}=0$ is however atypical and requires an independent Nyquist analysis.] With respect to the parameter $\eta_{i}$, two unstable ranges occur:

$$
\text { (i) } 0<\eta_{i}<\eta_{i, 1}=1-\sqrt{1-\zeta^{2} /\left(1+\tau_{i}\right)}
$$

(the case $\eta_{i}<0$ has not been investigated) and

$$
\text { (ii) } \eta_{i}>\eta_{i, 2}=1+\sqrt{1-\zeta^{2} /\left(1+\tau_{i}\right)} \text {. }
$$


[That result agrees with the standard ion temperature gradient instability criterion $\left(\eta_{i}>2\right.$ if $\left.\zeta=0\right)$.] At threshold, the wave frequency is

$$
\omega_{\mathrm{thr}}^{\prime}=\left(1-0.5 \eta_{i}\right) \omega_{e}^{*}
$$

$\left(\omega^{\prime}=\omega-\omega_{E}-k_{\|} U_{\|, i}\right.$ is the wave frequency in the ion rest frame) whereas the ratio of the parallel and binormal components of the wave vector obtained from Eq. $\left(36^{\prime}\right)$ is

$$
\left(k_{\|} / k_{\beta}\right)_{\mathrm{thr}}=\zeta a_{s} / 2\left(1+\tau_{i}\right) L_{N}=\partial_{r} U_{\|, i} / 2\left(1+\tau_{i}\right) \Omega_{i} .
$$

Equation (53) may be regarded as a generalized criterion for the parallel velocity shear instability and (55) as a generalized criterion for the ion temperature gradient instability. Equation (54) is rather related to the electron drift branch, as the frequency of the marginally stable mode $\omega_{\mathrm{thr}}^{\prime} \rightarrow \omega_{e}^{*}$ in the ion rest frame if $\zeta \rightarrow 0$. The frequency of the marginally stable ion drift mode $\omega_{\mathrm{thr}}^{\prime} \rightarrow 0^{+} \omega_{e}^{*}$ if $\zeta \longrightarrow 0 ; \omega_{\mathrm{thr}}^{\prime}$ has the sign of $\omega_{e}^{*}$ whatever the value of $\zeta \neq 0$.

(2) The growth/decay rate in the neighborhood of instability threshold is given by

$$
\operatorname{Im} \delta \omega^{\prime}=\left(1+\tau_{i}\right)\left(2 \frac{\delta \zeta}{\zeta_{\mathrm{thr}}}-\frac{(\delta \xi)^{2}}{\xi_{\mathrm{thr}}^{2}}\right) \frac{z_{\mathrm{thr}} \tau_{i} \mathfrak{I}}{\left(\mathfrak{R}-\tau_{i}\right)^{2}+\mathfrak{I}^{2}} \omega_{e}^{*},
$$

where $\mathfrak{R}$ and $\mathfrak{I}$ are given by $\left(46^{\prime}\right)$ and $\left(46^{\prime \prime}\right) ; \omega_{e}^{*}$ is positive by convention. The expansion leading to (58) is not valid if $\eta_{i} \rightarrow 2$, as $\zeta_{\text {thr }}, \xi_{\text {thr }}$, and $z_{\text {thr }}$ vanish in that limit.

(3) The components of the oscillating magnetic field are given in Eqs. (16), (51), and (52). We note that the perturbed parallel current arises as to cancel the charge separation which tends to arise in the perpendicular plane owing to finite ion gyroradius effects. The reason why $\beta$ enters neither the dispersion relation nor the stability criterion at the values being considered is presumably that we assumed $a_{i} \partial_{r}$ $\sim k_{\beta} a_{i} \sim \mu \ll 1 .^{14}$

\section{B. Justification of the approximations}

Equation (57) can be rewritten as

$$
\left(k_{\|} q R\right)_{\mathrm{thr}}=\left[\zeta / 2\left(1+\tau_{i}\right)\right] k_{\beta} a_{s}\left[q R / L_{N}\right] .
$$

We have estimated that $\zeta \sim 1$ and $q R / L_{N} \sim 6 \times 10^{2}$ in the pedestal of ALCATOR C-Mod H-mode discharges which are close to the ELM-free to EDA transition. ${ }^{5,16}$ Inserting the poloidal mode number $k_{\theta} \sim 400 \mathrm{~m}^{-1}$ of the QC mode observed in EDA discharges and $a_{i} \sim 0.35 \times 10^{-3} \mathrm{~m}$ (see Refs. 5 and 16) yields $\left(k_{\|} q R\right)_{\mathrm{thr}} \sim 20$. Since $k_{\|}=\mathbf{k} \cdot \mathbf{B} / B=(m$ $+n q) / q R$, we conclude that $m+n q \gg 1$ (where $m$ and $n$ are the poloidal and toroidal mode numbers). Under those conditions,

the poloidal and toroidal mode numbers $m$ and $n$ are independent [they are not linked by a relation of the type $\left.q\left(r_{m / n}\right)=-m / n\right]$;

(ii) the mode spans a large number $\left(=k_{\|} q R\right)$ of rational surfaces [those are defined by $q\left(r_{m / n^{\prime}}\right)=-m / n^{\prime}$ ];

(iii) the parallel ion dynamics cannot be balanced by finite ion gyroradius nor by curvature effects, which justifies the local approximation and the cylindrical model [parallel ion dynamics, ion gyroradius, and curvature yield terms proportional to $\left(k_{\|} c_{i} / \omega^{\prime}\right)^{2},\left(a_{i} \partial_{r}\right)^{2}$, and $\left(c_{i} a_{i} \partial_{r} / R \omega^{\prime}\right)^{2}$ in the wave equation; the ratios $\left(\omega^{\prime} / k_{\|} c_{i}\right) a_{i} \partial_{r} \sim\left(\omega^{\prime} / \omega_{i}^{*}\right)\left(L_{s} / L_{N}\right)\left(k_{\beta} a_{i} / k_{\|} L_{s}\right)^{2} \ll 1$ and $\left(a_{i} \partial_{r} / k_{\|} R\right) \sim k_{\beta} a_{i}(q / \hat{s}) /\left(k_{\|} L_{s}\right)^{2} \ll 1$ are here of interest (we approximate the radial wave number $\partial_{r}$ by $1 /\left(k_{\|} q R\right) \Delta, \Delta=1 / k_{\beta} \hat{s}$ being the distance between the neighboring rational surfaces $r_{m / n^{\prime}}$ and $r_{m \pm 1 / n^{\prime}}$; $\hat{s}=r \partial_{r} \ln q$ and $L_{s}=q R / \hat{s}$ are the magnetic shear parameter the magnetic shear length, respectively)];

(iv) the trapped electron response is negligible; ${ }^{17,18,19}$ hence, also in view of $\omega^{\prime} / k_{\|} c_{e} \ll 1$, the validity of the adiabatic approximation.

\section{Remarks}

(1) Since we have allowed the oscillations and equilibrium radial scales to be comparable and no term involving the radial gradients $\partial_{r}\left(n_{j}, p_{j}, \phi\right)$ appears in the dispersion equation, we conclude that the oscillations frequencies may be space dependent. For example $\omega=\left[1-\eta_{i}(r) / 2\right] \omega_{e}^{*}(r)$ $+\omega_{E}(r)+k_{\|} U_{\|, i}(r)$ for the marginally unstable mode.) As a result, a wave packet of size $w$ will get distorted and break up into smaller structures over a time interval $\tau=1 / w \partial_{r} \omega$. Since finite amplitude will be reached only if $\gamma \tau \geqslant 1$ (where $\gamma$ is the linear growth rate) the size of the wave packet is bounded by

$$
w \leqslant \gamma / \partial_{r} \omega
$$

The energy which is transferred to shorter radial wavelengths via the disintegration process might be reabsorbed through finite $\beta$ stabilization. ${ }^{14}$

The role of inhomogeneous electric fields and inhomogeneous parallel flows must here be contrasted. The stabilising role of the former can only be understood in the above context of wave packet distortion since its gradient does not enter the dispersion relation. The gradient of the parallel velocity not only affects the width of the wave packet (as it contributes to $\left.\partial_{r} \omega\right)$, but appears explicitly in the stability criterion and the expression of the linear growth rate. Those observations may explain the somewhat contrasted effects of the $\mathbf{E} \times \mathbf{B}$ velocity gradient and of the parallel velocity gradient which have been noticed in numerical simulations. ${ }^{20,21}$ We note finally that it is misleading, in our opinion, to singularize the stabilizing role of the $\mathbf{E} \times \mathbf{B}$ velocity gradient; the gradient of the diamagnetic frequencies, for example, should have similar consequences.

(2) If the width and the amplitude of the unstable wave packets are controlled by the frequency gradient, then energy, momentum, and particle bursts are expected when $\left|\partial_{r} \omega\right|$ decreases momentarily. [It is noted that small values of $\partial_{r} \omega / \omega$ do not necessarily require small values of $\partial_{r} T / T$ and $\partial_{r} N / N$ !.] That may be relevant to intermittent behavior. Conversely, $\partial_{r} \omega$ (and therefore the equilibrium gradients) will be controlled by anomalous transport; that is equivalent to self-organized criticality.

(3) It has been mentioned earlier (Sec. VII A) that reliable (in)stability criteria cannot be obtained from asymptotic 
expansions of the local dispersion relation. That certainly also applies for localized ITG-PVS modes, $\omega^{\prime} / k_{\|} c_{i}$ being again of order unity. Under those conditions, analytical progress will require us to either generalize the Nyquist technique or to be guided by results from gyrokinetic simulations. $^{22-24}$

(4) Equation (57) holds also above instability threshold for the central value of the parallel wave number in the unstable range (for a given $k_{\beta}$ ). The anomalous radial flow of parallel momentum will be proportional to a weighted value of $k_{\|}$over the spectrum and hence to $\partial_{r} U_{\|, i}$.

\section{ACKNOWLEDGMENTS}

The work of R.S. was partially financed by the Bundesministerium für Bildung und Forschung (BMBF) in the framework of the governmental agreement between the Republic of India and the government of the Federal Republic of Germany in cooperation in the fields of Scientific and Technology Development (Project No. IND 01/012).

\section{APPENDIX: THE DISPERSION RELATION FOR $\tau_{i} \rightarrow 0$ AND $\eta_{i} \rightarrow 0$}

The function $D\left(\omega^{\prime}\right)$ can be readily evaluated if $k_{\|} c_{i}$ $\ll \omega^{\prime}$, which limit always applies if $\tau_{i} \rightarrow 0$. As $\operatorname{Im} D\left(\omega^{\prime}\right)$ vanishes faster than any power of the small parameter $\tau_{i}$ [cf. Eq. (30)], the dispersion relation (28) takes the form

$$
z^{2}-z-\xi(\zeta+\xi)=0 .
$$

One of the roots is unstable if

$$
\xi^{2}+\zeta \xi+1 / 4 \leqslant 0
$$

i.e., if $\xi$ is in the range

$$
-\left(\zeta+\sqrt{\zeta^{2}-1}\right) \leqslant 2 \xi \leqslant-\left(\zeta-\sqrt{\zeta^{2}-1}\right) .
$$

That is possible if

$$
|\zeta|>\left|\zeta_{\text {thr }}\right|=1
$$

For $\zeta=\zeta_{\text {thr }}= \pm 1$, one has $\xi=\xi_{\text {thr }}=-\zeta_{\text {thr }} / 2$ and $z=z_{\text {thr }}=1 / 2$ (hence $\omega^{\prime}=\omega_{e}^{*} / 2$ ). Those results agree with those of Catto et al. but disagree with (36) and (37) unless $\eta_{i}=1$. The origin of the discrepancy has been discussed below Eq. (37) and at the end of Sec. V.
The dispersion relation near marginal stability is also readily obtained if $\eta_{i} \rightarrow 0$ and $\xi \propto \zeta \rightarrow 0$ [cf. (36) and $\left.\left(36^{\prime}\right)\right]$ : expanding for $k_{\|} c_{i} \ll \omega^{\prime}$ indeed yields $z=1$, in agreement with (37).

${ }^{1}$ M. Greenwald, R. Boivin, P. Bonoli et al., Phys. Plasmas 6, 1943 (1999).

${ }^{2}$ I. H. Hutchinson, R. Boivin, P. T. Bonoli et al., Nucl. Fusion 41, 1391 (2001).

${ }^{3}$ J. A. Snipes, B. LaBombard, M. Greenwald, I. H. Hutchinson, J. Irby, Y. Lin, A. Mazurenko, and M. Porkolab, Plasma Phys. Controlled Fusion 43, L23 (2001).

${ }^{4}$ N. D'Angelo, Phys. Fluids 8, 1748 (1965).

${ }^{5}$ A. L. Rogister, J. E. Rice, A. Nicolai, A. Ince-Cushman, S. Gangadhara, and ALCATOR C-Mod Group, Nucl. Fusion 42, 1144 (2002); see in particular Sec. 7.3.

${ }^{6}$ C. P. Perez, H. R. Koslowski, P. Smeulders et al., Plasma Phys. Controlled Fusion 46, 61 (2004).

${ }^{7}$ P. J. Catto, M. N. Rosenbluth, and C. S. Liu, Phys. Fluids 16, 1719 (1973).

${ }^{8}$ C. G. Smith and S. Von Goeler, Phys. Fluids 11, 2665 (1968).

${ }^{9}$ T. H. Stix, Waves in Plasmas (American Institute of Physics, New York, 1992), Sec. 8-11.

${ }^{10}$ R. J. Goldston and P. H. Rutherford, Introduction to Plasma Physics (Institute of Physics, Bristol, 1995), Sec. 26.6.

${ }^{11}$ S. I. Braginskii, in Reviews of Plasma Physics, edited by M. A. Leontovich (Consultants Bureau, New York, 1965), Vol. 1, p. 214.

${ }^{12}$ R. D. Hazeltine, Phys. Fluids 17, 961 (1974).

${ }^{13}$ H. A. Claassen, H. Gerhauser, A. Rogister, and C. Yarim, Phys. Plasmas 7, 3699 (2000).

${ }^{14}$ J. Q. Dong, P. N. Guzdar, and Y. C. Lee, Phys. Plasmas 30, 2694 (1987).

${ }^{15}$ A. Rogister and D. Li, Phys. Fluids B 4, 804 (1992), Appendix B.

${ }^{16}$ A. L. Rogister, "Enhanced D $\alpha$ confinement mode: A theoretical model," Nucl. Fusion (submitted).

${ }^{17}$ A. L. Rogister and G. Hasselberg, "Asymptotic theory of the dissipative trapped electron mode overlapping many rational surfaces," in Proceedings of the Varenna Workshop 1977 (Pergamon, Oxford, 1978), p. 49.

${ }^{18}$ P. J. Catto and K. T. Tsang, Phys. Fluids 21, 1381 (1978).

${ }^{19}$ A. L. Rogister and G. Hasselberg, Phys. Fluids 22, 2382 (1979).

${ }^{20}$ A. M. Dimits, B. I. Cohen, W. M. Nevins, and D. E. Shumaker, Nucl. Fusion 41, 1725 (2001).

${ }^{21}$ C. G. Hobbs, M. G. House, J. N. Leboeuf, J. M. Dawson, V. K. Decyk, M. W. Kissik, and R. D. Sydora, Phys. Plasmas 8, 4849 (2001).

${ }^{22}$ W. Dorland, B. N. Rogers, F. Jenko et al., Fusion Energy 2000, in Proceedings of the 18th International Conference, Sorrento, 2000 (IAEA, Vienna, 2001), CD-ROM, file TH2/5 and http://www.iaea.org/ programmes/ripc/physics/fec2000/html/node1.htm

${ }^{23}$ F. Jenko and W. Dorland, Plasma Phys. Controlled Fusion 43, A141 (2001).

${ }^{24}$ D. W. Ross and W. Dorland, Phys. Plasmas 9, 5031 (2002). 